\title{
Epidermal growth factor enhances MPC-83 pancreatic cancer cell migration through the upregulation of aquaporin 3
}

\author{
WEIJUN LIU ${ }^{1 *}$, KUNHUA WANG ${ }^{1 *}$, KUNMEI GONG ${ }^{1}$, XIAOGANG LI $^{2}$ and KAIYUAN LUO ${ }^{2}$ \\ ${ }^{1}$ Department of General Surgery, Kunhua Hospital Affiliated to Kunming Medical College, Kunming, Yunnan 650032; \\ ${ }^{2}$ Department of General Surgery, the Fourth Affiliated Hospital of Kunming Medical College, \\ Kunming, Yunnan 650021, P.R. China
}

Received March 18, 2012; Accepted June 13, 2012

DOI: $10.3892 / \mathrm{mmr} .2012 .966$

\begin{abstract}
Aquaporin (AQP) water channels are expressed in high-grade tumor cells of different tissue origins. In this study, we investigated whether AQP3 is expressed in cultured MPC-83 pancreatic cancer cells, whether AQP3 enhances cell migration and the signal pathway mechanism involved. MPC-83 pancreatic cancer cells were pre-treated and treated with EGF at different time points and then analyzed using western blotting. Results showed that epidermal growth factor (EGF) induced the phosphorylation of the EGF receptor (EGFR) and extracellular signal-regulated kinase (ERK), which peaked at $5 \mathrm{~min}$ after EGF treatment. EGFR and ERK phosphorylation induced by EGF were inhibited by PD153035 (EGFR tyrosine kinase inhibitor) and U0126 (ERK inhibitor), respectively. EGF increased the activity of AQP3 in a doseand time-dependent manner in MPC-83 pancreatic cancer cells, which peaked at $24 \mathrm{~h}$ after treatment. The activity of AQP3 and cell migration were inhibited by PD153035, U0126 and $\mathrm{CuSO}_{4}$ (AQP3 water transport inhibitor). EGFR/ERK pathway-mediated AQP3 activation and cell migration were stimulated by EGF in cultured MPC- 83 pancreatic cancer cells in vitro and this cell signaling pathway is inhibited by the EGFR and ERK inhibitors, which may be used as potential therapeutic targets in the treatment of pancreatic cancer.
\end{abstract}

\section{Introduction}

Aquaporins (AQPs) are small, integral membrane proteins that selectively transport water across cell plasma membranes.

Correspondence to: Dr Kaiyuan Luo, Department of General Surgery, the Fourth Affiliated Hospital of Kunming Medical College, 176 Qingnian Avenue, Wuhua District, Kunming, Yunnan 650021, P.R. China

E-mail: liu1350871@yahoo.cn

*Contributed equally

Key words: aquaporin 3, pancreatic cancer cell, cell migration, epidermal growth factor receptor
AQPs are expressed in a number of fluid-transporting tissues, including kidney tubules and glandular epithelia, as well as in non-fluid-transporting tissues, such as epidermis, adipose tissue and astroglia. AQPs are strongly expressed in tumor cells of different origins, particularly aggressive tumors (1). AQP expression in tumors has been suggested to be of diagnostic and prognostic value. Recent discoveries of AQP involvement in cell migration and proliferation suggest that AQPs play key roles in tumor biology. AQP-dependent cell migration has been found in a variety of cell types in vitro and in mice in vivo. AQP3 has been found to be expressed in cultured fibroblasts, with AQP3 knockdown by RNA inhibition reducing fibroblast migration (2). Impaired cell migration has also been observed in AQP1-deficient proximal tubule epithelial cells, AQP3-deficient corneal epithelial cells and enterocytes (3).

The epidermal growth factor (EGF) family of growth factors exert their roles and elicit a series of physiological and pathological actions by binding to the EGF receptor (EGFR) family. Activation of the EGFR family has been identified as a key event that initiates the cascade of intracellular signaling pathways leading to proliferation, cell survival, angiogenesis and metastasis (4). A number of the most common human epithelial cancers express relatively high levels of EGFR at advanced stages of malignancy and an increased metastatic potential of the disease (5). Elevated expression or activity of EGFR is common in human cancer and is associated with poor patient prognosis (6). Therefore, we hypothesized that AQPs in MPC-83 pancreatic cancer cells also mediate EGF-induced cell migration and thus their metastatic potential.

In the present study, we investigated whether EGF induced the phosphorylation of EGFR and ERK and if so, whether AQP3 expression was upregulated by EGF. We also observed whether EGF facilitated MPC-83 pancreatic cancer cell migration. Using western blot analysis and in vitro cell migration, we found that EGF phosphorylates EGFR and ERK and that AQP3 expression is upregulated by EGF. EGF, via the EGFR signaling pathway, facilitated AQP3 expression, which is involved in cell migration in MPC-83 pancreatic cancer cells. Our findings provide an explanation as to the molecular mechanisms of MPC-83 pancreatic cancer cell migration and may contribute to potential therapeutic strategies for the treatment of pancreatic cancer. 


\section{Materials and methods}

Reagents. Rabbit anti-aquaporin 3 was obtained from Chemicon (Temecula, CA, USA). Rabbit anti-phospho-EGFR (Tyr1068), phospho-ERK and ERK were obtained from Cell Signaling Technology (Beverly, MA, USA). Rabbit anti-mouse IgG-HRP antibody was obtained from Santa Cruz Biotechnology, Inc. (Santa Cruz, CA, USA). Monoclonal mouse anti- $\beta$-actin was obtained from Sigma (St. Louis, MO, USA). PD153035 and U0126 were obtained from CalbioChem (San Diego, CA, USA). $\mathrm{CuSO}_{4}$ was obtained from Sigma.

Cell culture. Cultured pancreatic cancer cell line MPC-83 cells were maintained in DMEM (Sigma) supplemented with $10 \%$ fetal bovine serum (FBS), penicillin/streptomycin (1:100, Sigma) and $4 \mathrm{mM}$ L-glutamine (1:100, Sigma), in a $\mathrm{CO}_{2}$ incubator at $37^{\circ} \mathrm{C}$. For western blot analysis, cells were reseeded in 6-well plates at a density of $0.2 \times 10^{6}$ cells $/ \mathrm{ml}$ with fresh complete culture medium. Morphological changes were observed under a phase contrast microscope.

Western blot analysis. Cultured MPC-83 pancreatic cancer cells with or without treatment were washed with cold PBS and harvested by scraping into $150 \mu \mathrm{l}$ of RIPA buffer (containing $50 \mathrm{mM}$ Tris- $\mathrm{HCl}$, pH 7.4, $150 \mathrm{mM} \mathrm{NaCl}, 1 \% \mathrm{NP} 40$, $1 \mathrm{mM}$ EDTA, $0.25 \%$ sodium deoxycholate) with fluoride and protease inhibitor cocktail ( $10 \mu \mathrm{l} / \mathrm{ml}$ leupeptin, $10 \mu \mathrm{l} / \mathrm{ml}$ aprotinin and $1 \mu \mathrm{M}$ pepstatin). Cell lysates were incubated at $4^{\circ} \mathrm{C}$ for $30 \mathrm{~min}$. After centrifugation at $14,000 \mathrm{rpm}$ for $10 \mathrm{~min}$ at $4^{\circ} \mathrm{C}$, the protein concentration was determined by a Bio-Rad protein assay (Bio-Rad, Hercules, CA, USA). Proteins $(20 \mu \mathrm{g}$ for AQP3, phospho-EGFR and EGFR, phospho-ERK and ERK; $10 \mu \mathrm{g}$ for $\beta$-actin) were denatured in 5X SDS-PAGE sample buffer for $5 \mathrm{~min}$ at $95^{\circ} \mathrm{C}$. The proteins were separated by 10 or $7.5 \%$ SDS-PAGE and transferred onto PVDE membranes (Millipore, Bedford, MA, USA) for $2 \mathrm{~h}$ at $4^{\circ} \mathrm{C}$. Non-specific binding was blocked with $10 \%$ dry milk in TBST (20 mM Tris- $\mathrm{HCl}, 137 \mathrm{mM} \mathrm{NaCl}, 0.01 \%$ Tween-20, $\mathrm{pH}$ 7.4) for $1 \mathrm{~h}$ at room temperature. After blocking, the membranes were incubated with specific antibodies against AQP3 $(1: 1,000)$, EGFR $(1: 1,000)$, phospho-EGFR $(1: 1,000)$, phospho-ERK and ERK $(1: 1,000)$ and $\beta$-actin $(1: 20,000)$ in dilution buffer (2\% BSA in TBS) overnight at $4^{\circ} \mathrm{C}$. The blots were incubated with HRP-conjugated anti-rabbit or antimouse IgG at appropriate dilutions and room temperature for $1 \mathrm{~h}$. Antibody binding was detected using an enhanced chemiluminescence (ECL) detection system following the manufacturer's instructions and visualized by autoradiography with Hyperfilm.

Phagokinetic track motility assay. Twelve-well plates were coated with coating medium of $20 \mu \mathrm{g} / \mathrm{ml}$ fibronectin (Sigma) in PBS and placed in a $\mathrm{CO}_{2}$ incubator at $37^{\circ} \mathrm{C}$ for at least $2 \mathrm{~h}$. After removing the coating medium gently with a pasteur pipette, the wells were washed with PBS and $2.4 \mathrm{ml}$ of microsphere suspension ( $86 \mu \mathrm{l}$ of stock microbeads solution in $30 \mathrm{ml}$ PBS) was added to each well. The plates were then centrifuged at $1,200 \mathrm{rpm}$ at $4^{\circ} \mathrm{C}$ for $20 \mathrm{~min}$ and carefully transferred to a $\mathrm{CO}_{2}$ incubator and incubated at $37^{\circ} \mathrm{C}$ for at least $1 \mathrm{~h}$. From each well, $1.8 \mathrm{ml}$ of supernatant was removed and
1,500 freshly trypsinized cells in $2 \mathrm{ml}$ assay medium (DMEM supplemented with a $0.05 \%$ FBS) were seeded per well. The cells were cultured for $24 \mathrm{~h}$ and photographed under a phase contrast microscope.

Statistical analysis. Experiments were performed in triplicate. Data were presented as the mean \pm standard error (SE). Comparisons among groups were performed using analysis of variance (ANOVA). $\mathrm{P}<0.05$ was considered to indicate statistically significant differences.

\section{Results}

Phosphorylation of EGFR and ERK was induced by EGF in MPC-83 pancreatic cancer cells. MPC-83 pancreatic cancer cells were pre-treated with EGF at different time points, then p-EGFR and p-ERK were detected by western blotting. The results showed that EGFR phosphorylation was induced by EGF. This phosphorylation peaked at $5 \mathrm{~min}$ and lasted $1 \mathrm{~h}$ (Fig. 1A). Similar results were observed in ERK phosphorylation, which peaked at $5 \mathrm{~min}$ after EGF treatment and lasted 40 min (Fig. 1B). The phosphorylation of EGFR and ERK induced by EGF was significantly inhibited in MPC-83 pancreatic cancer cells which were pre-treated by PD153035 (PD) or U0126 (U0) (Fig. 1C and D). This finding confirms that EGF induces the phosphorylation of EGFR and further activates its downstream signal pathway.

Expression of AQP3 was upregulated by EGF in MPC-83 pancreatic cancer cells. Cells were treated with EGF at different concentrations of 10,50 and $100 \mathrm{ng} / \mathrm{ml}$ and cell lysates were analyzed for AQP3 by western blotting. The results showed that EGF induced the upregulation of AQP3 in a dose-dependent manner in MPC-83 pancreatic cancer cells (Fig. 2A). AQP3 expression begins to increase after cells are treated with $50 \mathrm{ng} / \mathrm{ml}$ of EGF and is highest at $100 \mathrm{ng} / \mathrm{ml}$ of EGF treatment. The expression of AQP3 was upregulated by EGF in a time-dependent manner (Fig. 2B). AQP3 begins to increase at $12 \mathrm{~h}$ and is most obvious at $24 \mathrm{~h}$ after being treated with $100 \mathrm{ng} / \mathrm{ml}$ of EGF. The expression of AQP3 was inhibited in MPC-83 pancreatic cancer cells which were pre-treated by PD or U0 followed with EGF treatment (Fig. 2C). The data show the mean $\pm \mathrm{SE}$ of triplicate experiments.

Migration of MPC-83 pancreatic cancer cells was inhibited by $\mathrm{PD}, \mathrm{UO}$ and $\mathrm{CuSO}_{4}$ (water and glycerol transport inhibitors of AQP3). The migration of MPC-83 pancreatic cancer cells which were pre-treated with $\mathrm{PD}, \mathrm{U} 0$ and $\mathrm{CuSO}_{4}$ followed by EGF treatment was significantly inhibited (Fig. 3).

\section{Discussion}

Pancreatic cancer remains a potentially fatal malignancy giving rise to continuing concern for a number of reasons. Pancreatic cancer is a major unsolved health problem, with conventional cancer treatments having little impact on disease course. Almost all patients who have pancreatic cancer develop metastases and succumb to the disease. The main risk factors are smoking, age and certain genetic disorders, although the primary causes are poorly understood. 
A
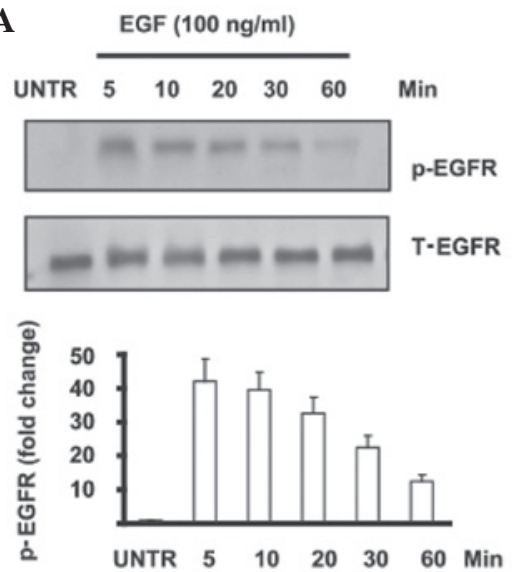

C
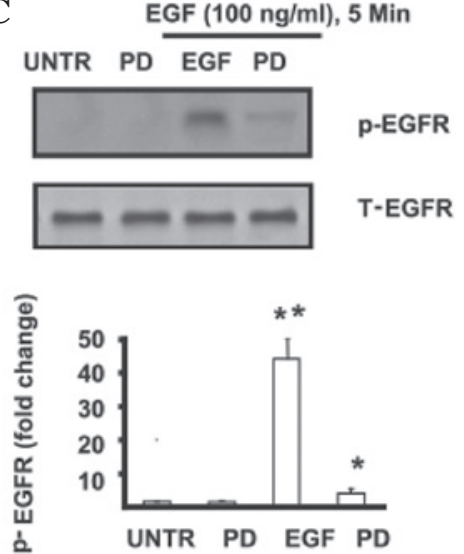

B
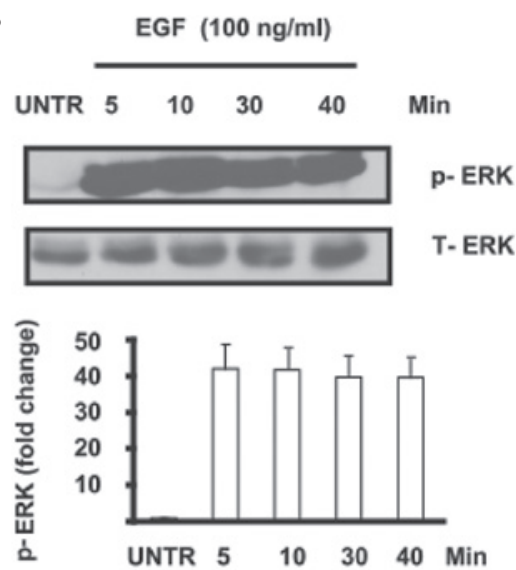

D
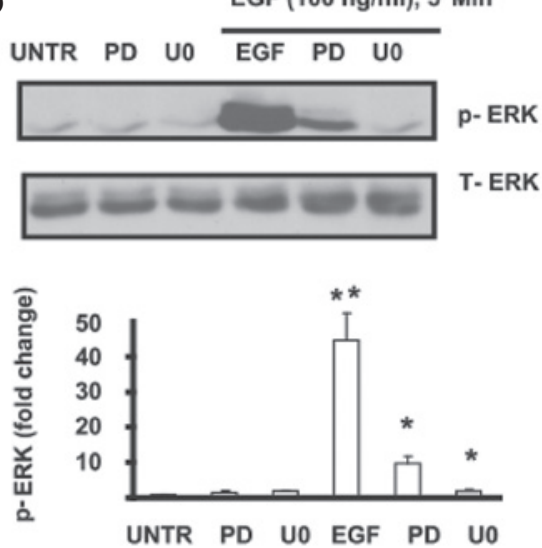

Figure 1. (A) Cells were treated with EGF (100 ng/ml) and collected at different time points (5, 15, 30 and 60 min). EGFR phosphorylation was analyzed by western blotting and quantified. (B) Cells were treated with EGF $(100 \mathrm{ng} / \mathrm{ml})$ and collected at different time points (5, 10, 30 and $40 \mathrm{~min})$, cell lysates were collected and ERK phosphorylation was analyzed by western blotting and quantified. (C) Cells were pre-treated with or without PD (PD153035) followed by EGF (100 ng_ml) for $5 \mathrm{~min}$, and EGFR phosphorylation was analyzed by western blotting and quantified. (D) Cells were pre-treated with or without PD or U0 (U0126) followed by EGF (100 ng/ml) for 5 min, and ERK phosphorylation was analyzed by western blotting and quantified. The data are presented as the mean \pm SE of triplicate experiments. ${ }^{*}<<0.05$. EGF, epidermal growth factor; EGFR, EGF receptor; ERK, extracellular signal-regulated kinase.

A
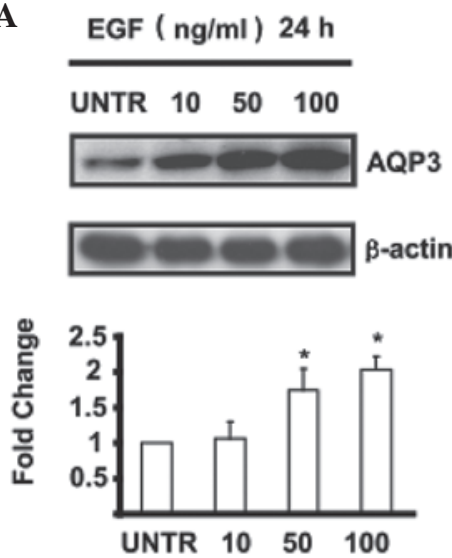

B
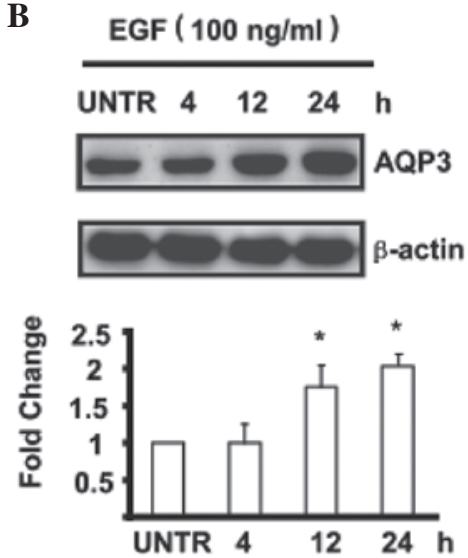

C
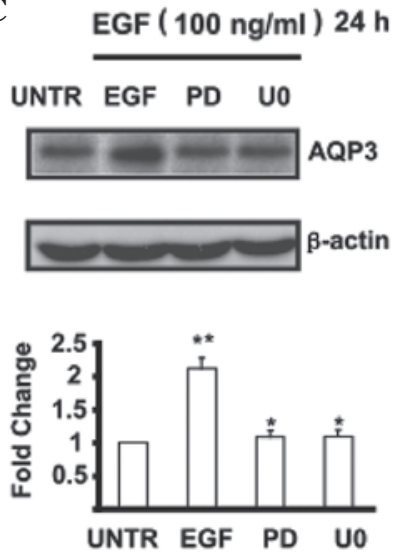

Figure 2. (A) Cells were treated with various doses of EGF (10,50, $100 \mathrm{ng} / \mathrm{ml})$ and collected at $24 \mathrm{~h}, \mathrm{AQP} 3$ expression was analyzed by western blotting and quantified. (B) Cells were treated with EGF (100 ng/ml) and collected at different time points (4,12 and $24 \mathrm{~h})$, AQP3 expression was analyzed by western blotting and quantified. (C) Cells were pre-treated with or without PD (PD153035) or U0 (U0126) followed by EGF (100 ng/ml) for 24 h, AQP expression was analyzed by western blotting and quantified. EGF, epidermal growth factor; AQP, aquaporin.

Tumor-committed cells generally have an aggressive metabolic energy profile, allowing them to compete with surrounding cells, proliferate and form characteristic struc- tures. At least 12 different tumor cell types have been reported to express AQPs in vivo in humans and rodents. For certain tumors, positive correlations have been established between 
EGF ( $100 \mathrm{ng} / \mathrm{ml}) 24 \mathrm{~h}$
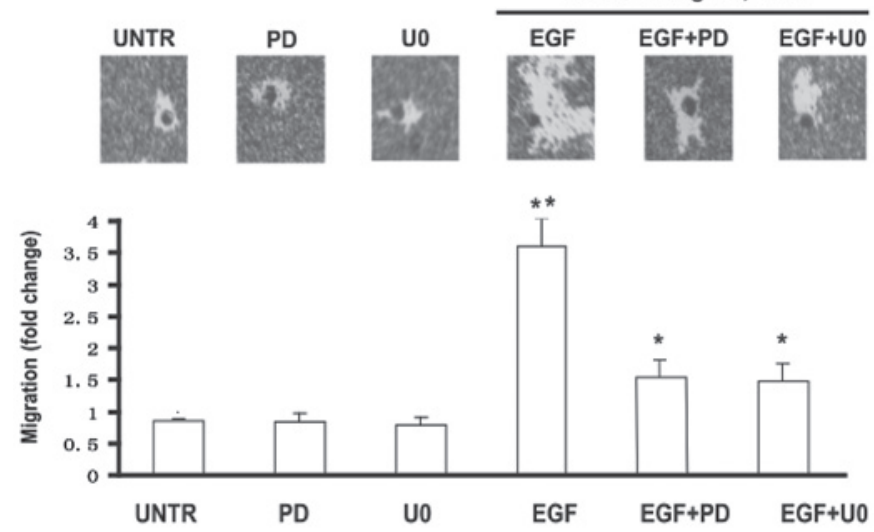

Figure 3. Cells were treated with EGF (100 ng/ml) and inhibitors (PD153035, $\mathrm{U} 0126$ or $\mathrm{CuSO}_{4}$ ). Cell migration was inhibited and photographed at $24 \mathrm{~h}$. EGF-induced cell migration was inhibited by PD153035, U0126 or $\mathrm{CuSO}_{4}$ in triplicate experiments. ${ }^{*} \mathrm{P}<0.05$. EGF, epidermal growth factor.

histological tumor grade and the level of AQP expression. AQP3, which is found in normal epidermis and becomes upregulated in basal cell carcinoma, facilitates cell proliferation in different cell types. AQP3-null mice are resistant to skin tumorigenesis by a mechanism that may involve reduced tumor cell glycerol metabolism and ATP generation (7). AQP expression in tumors is known to facilitate tumor cell migration and spread, suggesting a novel function for AQP expression in high-grade tumors. One consequence of AQP involvement in tumor cell migration is the possibility of AQP inhibition to limit tumor spread, although testing of this possibility requires the development of suitable AQP-selective inhibitors (8). We have found that AQP3 is expressed in cultured MPC-83 pancreatic cancer cells. Using specific AQP3 water transport inhibitors, it is confirmed that AQP3 is involved in EGF-induced pancreatic cancer cell migration in vitro.

Growth factors control cell growth, proliferation, differentiation, survival and migration by activating receptors on specific target cells. Aberrant activation of EGFR and the EGF signal pathway is associated with neoplastic cell proliferation, migration, stromal invasion, resistance to apoptosis and angiogenesis (9). Aberrant EGFR expression and signaling contribute to the development of multiple epithelial malignancies in humans, including squamous cell carcinomas of the skin and breast cancer (10). In particular, EGFR expression is upregulated in $33-50 \%$ of human epithelial tumors (11). In this study, we confirm that AQP3 upregulation is involved in MPC-83 pancreatic cancer cell migration and this effect can be induced by EGF. We also provide evidence that an EGFR-mediated MEK/ERK pathway is involved in EGF-induced AQP3 expression and cell migration in MPC-83 pancreatic cancer cells.
EGFR-mediated signaling pathways have been shown to contribute to the regulation of angiogenesis and metastasis (12). To demonstrate whether one of these pathways was involved in MPC-83 pancreatic cancer cell migration, EGFR and ERK inhibitors were used. We found that these two inhibitors suppressed AQP3 expression and cell migration induced by EGF in MPC-83 pancreatic cancer cells. The above results clearly demonstrate that an EGF/EGFR/ERK signaling pathway is at least partly involved in EGF-induced AQP3 expression and cell migration in MPC-83 pancreatic cancer cells. First, EGF induces the phosphorylation of EGFR and ERK, then AQP3 expression is up-regulated and finally, MPC-83 pancreatic cancer cell migration is enhanced.

We conclude that AQP3 is expressed in MPC-83 pancreatic cancer cells and AQP3 facilitates MPC-83 pancreatic cancer cell migration. EGF upregulates AQP3 expression and cell migration via the EGFR/ERK signal transduction pathway in MPC-83 pancreatic cancer cells. This is likely to aid in future efforts to target appropriate EGFR-mediated signals as a rational cancer therapy.

\section{References}

1. Jeyaseelan K, Sepramaniam S, Armugam A, et al: Aquaporins: a promising target for drug development. Expert Opin Ther Targets 10: 889-909, 2006.

2. Walz T, Fujiyoshi Y and Engel A: The AQP structure and functional implications. Handb Exp Pharmacol: 31-56, 2009.

3. Verkman AS, Hara-Chikuma $M$ and Papadopoulos MC: Aquaporins-new players in cancer biology. J Mol Med (Berl) 86: 523-529, 2008.

4. Sartore-Bianchi A, Bencardino K, Di Nicolantonio F, et al: Integrated molecular dissection of the epidermal growth factor receptor (EFGR) oncogenic pathway to predict response to EGFR-targeted monoclonal antibodies in metastatic colorectal cancer. Target Oncol 5: 19-28, 2010.

5. Perol $M$ and Arpin D: Tyrosine kinase inhibitors in the management of non-small cell lung cancer. Rev Mal Respir 24: 6S188-6S197, 2007.

6. Valentini AM,Pirrelli M and Caruso ML: EGFR-targeted therapy in colorectal cancer: does immunohistochemistry deserve a role in predicting the response to cetuximab? Curr Opin Mol Ther 10: 124-131, 2008.

7. Nico B and Ribatti D: Role of aquaporins in cell migration and edema formation in human brain tumors. Exp Cell Res 317: 2391-2396, 2011.

8. Papadopoulos MC, Saadoun S and Verkman AS: Aquaporins and cell migration. Pflugers Arch 456: 693-700, 2008.

9. Giaccone G and Wang Y: Strategies for overcoming resistance to EGFR family tyrosine kinase inhibitors. Cancer Treat Rev 37: 456-464, 2011.

10. Mahipal A, McDonald MJ, Witkiewicz A, et al: Cell membrane and cytoplasmic epidermal growth factor receptor expression in pancreatic ductal adenocarcinoma. Med Oncol 29: 134-139.

11. Steelman LS, Franklin RA, Abrams SL, et al: Roles of the Ras/ Raf/MEK/ERK pathway in leukemia therapy. Leukemia 25: 1080-1094, 2011.

12. Dragovich T and Campen C: Anti-EGFR-targeted therapy for esophageal and gastric cancers: an evolving concept. J Oncol: $804108,2009$. 